\title{
Intense Slow Muon Physics
}

\author{
Yoshitaka Kuno a \\ ${ }^{a}$ Department of Physics, Osaka University, Toyonaka, Osaka 560-0043, Japan
}

Physics programs with slow muons at a neutrino factory are described. Emphasis is given to the muon trio, the muon $g-2$ anomalous magnetic moment, the muon electric dipole moment (EDM) and charged lepton mixing (CLM). In particular, a future muon source with high-intensity and high-luminosity, PRISM, developed at Osaka University is described.

\section{Muon Trio}

An overview (but not comprehensive) list of intense slow muon physics programs at a future neutrino factory, ranging from particle physics, nuclear physics to applications, is given in Table 1 , where programs are categorized into three groups, (1) precision measurements, (2) search for rare processes, and (3) application.

Among the particle physics programs with muons [1], very important are (a) precision measurement of the muon $g-2$ anomalous magnetic moment, (b) search for T-odd muon electric dipole moment (EDM), and (c) search for charged lepton mixing $(\mathrm{CLM})^{1}$. I call them "the muon trio". They are significantly important to search for new physics, and also closely related one another in some cases of new physics, such as supersymmetry (SUSY). From this reason, I would focus on "the muon trio" hereafter.

The goals of next generation experiments on the muon trio might be given as follows.

$$
\begin{array}{ll}
\text { Muon } g-2 & 0.5 \mathrm{ppm} \rightarrow 0.05 \mathrm{ppm} \\
\text { Muon EDM } & 10^{-19} e \cdot \mathrm{cm} \rightarrow 10^{-24} e \cdot \mathrm{cm} \\
\text { Muon CLM } & B\left(\mu^{-}+N \rightarrow e^{-}+N\right)<10^{-18}
\end{array}
$$

The physics motivation of the muon trio should be robust for next decade. In the following, the physics cases are discussed for two possible cases on LHC, namely whether LHC finds SUSY (Case I) or does not (Case II).

\footnotetext{
${ }^{1}$ It is lepton flavor violation for charged leptons.
}

\section{Case I: LHC finds SUSY.}

It is known that all the muon trio has significant contributions from SUSY, if SUSY particles exist in the LHC mass range. In this case, studies of the muon trio would focus on SUSY. In SUSY models, SUSY contributions can be presented by a slepton mass matrix, given in Eq. (1).

$$
\text { slepton mass matrix }=\left(\begin{array}{c}
m_{\tilde{e} \tilde{e}}^{2}, \Delta m_{\tilde{e} \tilde{\mu}}^{2}, \Delta m_{\tilde{e} \tilde{\tau}}^{2} \\
\Delta m_{\tilde{\mu} \tilde{e}}^{2}, m_{\tilde{\mu} \tilde{\mu}}^{2}, \Delta m_{\tilde{\mu} \tilde{\tau}}^{2}
\end{array}\right)(1)
$$

The SUSY contributions to the muon $g-2$, muon EDM, and muon CLM are a real part of the diagonal element $m_{\tilde{\mu} \tilde{\mu}}^{2}$, an imaginary part of the same diagonal element $m_{\tilde{\mu} \tilde{\mu}}^{2}$, and an off-diagonal element $\Delta m_{\tilde{\mu} \tilde{e}}^{2}$ or $\Delta m_{\tilde{e} \tilde{\mu}}^{2}$, respectively. Therefore, determination of these three SUSY contributions would enable us to study the slepton mass matrix and therefore SUSY (soft) breaking. In the following, the SUSY contributions for each of the muon trio can be described.

\subsection{Muon $g-2$}

The E821 at Brookhaven National Laboratory (BNL) carried out precision measurements of the anomalous $g-2$ magnetic moments of $\mu^{+}$and $\mu^{-}$. Their combined result is [2]

$a_{\mu}=\frac{g-2}{2}=11659208(6) \times 10^{-10} \quad(0.5 \mathrm{ppm})$

There is a difficulty in estimation of the standard model (SM) value of the muon $g-2$, since the hadronic contribution has a large uncertainty, 
Table 1

Overview list of slow muon physics programs.

\begin{tabular}{llll}
\hline Categories & Topics & Comments & Beam \\
\hline Precision measurements & muon lifetime & $G_{F}$ determination & pulsed \\
& muon capture rates & nuclear physics & pulsed or DC \\
& muonic X-ray & nuclear physics & pulsed or DC \\
& muon $g-2$ & SM allowed, new physics & pulsed \\
\hline Rare processes & muon EDM & SM suppressed, new physics & pulsed \\
& charged lepton mixing & SM forbidden, new physics & pulsed or DC \\
\hline Application & catalyzed fusion & aim break-even & pulsed \\
& materials science & $\mu \mathrm{SR}$ & DC \\
\hline
\end{tabular}

depending on which experimental data are used. If the data from $e^{+} e^{-}$collision experiments are used, a deviation from the SM prediction is $\Delta a_{\mu}=(23.9 \pm 9.9) \times 10^{-10}$ yielding $2.6 \sigma$ level, whereas when the data from $\tau$ decays are used, $\Delta a_{\mu}=(7.6 \pm 8.9) \times 10^{-10}$, yielding $0.9 \sigma$ level.

On the other hand, the SUSY contribution to the muon $g-2$ is given as follows,

$a_{\mu}^{\mathrm{SUSY}} \sim 13 \times 10^{-10} \cdot \tan \beta \cdot\left(\frac{100 \mathrm{GeV}}{\tilde{m}}\right)^{2}$

From Eq.(3), the possible deviation can be explained by SUSY contribution with its right magnitude, if $\tan \beta$ and a typical SUSY mass $\tilde{m}$ are in the $\mathrm{LHC}$ range.

In near future, a new experiment BNL-E969 to improve the muon $g-2$ value down to 0.2 ppm was scientifically approved, and is waiting for funding. In further future, a letter of intent to J-PARC, Japan, to improve down to $0.05 \mathrm{ppm}$ with higher muon beam intensity and the use of backward-decay muons.

\subsection{Muon EDM}

The muon $\operatorname{EDM}\left(d_{\mu}\right)$ is T and P odd quantity. The SUSY contributions to the muon EDM and the muon $g-2$ are closely related, since they are an imaginary and a real parts of the same diagrams of new physics. A naive estimation of new physics contribution to the muon $\operatorname{EDM}\left(d_{\mu}^{N P}\right)$ is made as follows [3],

$d_{\mu}^{N P} \sim 3 \times 10^{-22}\left(\frac{\Delta a_{\mu}^{N P}}{3 \times 10^{-9}}\right) \tan \phi_{C P}$

where $\phi_{C P}$ is an unknown CP imaginary phase, and $\Delta a_{\mu}^{N P}$ is new physics contribution to the muon $g-2$. From Eq.(4), the muon EDM of an order of $10^{-24} e \cdot \mathrm{cm}$ at the maximum can be possible for a large $\phi_{C P}$.

In minimal SUSY seesaw models consistent with neutrino oscillation data, $d_{\mu}$ has been also estimated, assuming CP violation in soft SUSY breaking parameters induced neutrino Yukawa couplings. The prediction ranges in $10^{-26}-$ $10^{-28} e \cdot \mathrm{cm}$, but it could become larger for some parameters. It is also noticed that in SUSY models, a ratio of the muon EDM to the electron EDM, $d_{\mu} / d_{e}$, may not be proportional to their mass ratio, $m_{\mu} / m_{e}$, and could be enhanced more for some parameter cases.

\subsection{Charged Lepton Mixing with Muons}

Neutrino mixing has been experimentally confirmed by discovery of neutrino oscillation. Now, lepton flavor conservation is known to be violated. However, charged lepton mixing (CLM) has never been observed yet experimentally. It is known that the contribution of neutrino mixing to charged lepton mixing is extremely small, since it is proportional to $\left(m_{\nu} / m_{W}\right)^{4}$, yielding the order of $10^{-50}$ in branching ratio. Therefore, discovery of charged lepton mixing would imply new physics beyond "neutrino oscillation".

In minimum SUSY models, charged lepton mixing would occur through mixing of their corresponding sleptons. Fig.1 shows one of the SUSY diagrams contributing to muon to electron transition, where the mixing of smuon $(\tilde{\mu})$ and selectron $(\tilde{e})$ is given by an off-diagonal slepton mass matrix $\Delta m_{\tilde{\mu} \tilde{e}}^{2}$. In minimum SUSY models, the slepton mass matrix is assumed to be a 


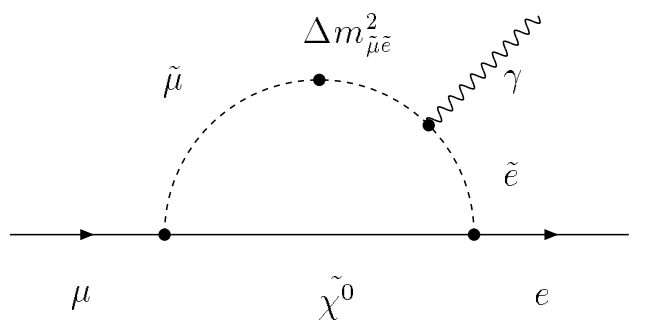

Figure 1. One of diagrams of SUSY contribution to a $\mu$ to $e$ transition. $\Delta m_{\tilde{\mu} \tilde{e}}^{2}$ indicates the magnitude of that slepton mixing.

diagonal matrix at the Planck mass scale $\left(10^{19}\right.$ $\mathrm{GeV}$ ), and no off-diagonal matrix elements exits $\left(\Delta m_{\tilde{\mu} \tilde{e}}^{2}=0\right)$. Then, non-zero off-diagonal matrix elements can be induced by radiative corrections from the Planck scale to the weak scale $\left(\sim 10^{2}\right.$ $\mathrm{GeV}$ ). There could be two scenarios at high energy to induce off-diagonal elements of the slepton mass matrix. One is grand unification (GUT) where the GUT Yukawa interaction creates nonzero off-diagonal elements. This scenario is called "SUSY-GUT" models. And the other is Seesaw mechanism, where the neutrino Yukawa interaction does. This is called "SUSY-Seesaw" models. Both of the models predict the branching ratios of CLM from just below to a few orders of magnitude below the current experimental upper limits. Therefore, if we could improve experimental sensitivity by a few orders of magnitude, this would provide great discovery potential.

When LHC finds SUSY, charged lepton mixing attract more interest on studies of either SUSYGUT) or SUSY-Seesaw models, rather than just minimum SUSY. There are not many ways to study them. In particular, to my knowledge, charged lepton mixing is only the way to study the Seesaw mechanism via SUSY.

\subsubsection{P-odd and T-odd asymmetries}

If CLM is discovered, a next step to study on CLM is P-odd asymmetry [1]. By using polarized muons, angular distribution of the electron produced by CLM with respect to the muon spin vector can be measured, whether it follows $1+\cos \theta_{e}$ or $1-\cos \theta_{e}$, where $\theta_{e}$ is an angle between the muon spin direction and the direction of electron moving. This would tell us the chiral- ity of the slepton that causing the mixing. This would be useful to discriminate whether SUSYGUT SU(5), SUSY-GUT SO(10), SUSY-Seesaw models or others.

T-odd asymmetry in CLM might also be ambitiously tried to measure. One of the examples is a T-odd correlation such $\vec{s}_{\mu} \cdot\left(\vec{p}_{e^{+}} \times \vec{p}_{e^{-}}\right)$in $\mu^{+} \rightarrow e^{+}+e^{+}+e^{-}$decay, where $\vec{s}_{\mu}$ is a muon spin vector, and $\vec{p}$ is a momentum vector. This T-odd asymmetry is expected to be up to $15 \%$.

\section{Case II: LHS does not find SUSY}

When LHC does not find SUSY, two cases can be considered; no SUSY or heavier SUSY at multi $\mathrm{TeV}$ scale. High precision frontier with intense slow muons comes to the forefront, since it is sensitive to heavier mass scale than that high-energy accelerators can reach. For instance, muon $g-2$ can be sensitive to the muon substructure that is given by $\Delta a_{\mu} \sim m_{\mu}^{2} / \Lambda_{\mu}^{2}$, or anomalous $W \gamma \gamma$ couplings. For the muon CLM, besides SUSY, there are other models to predict CLM, such as heavy neutrino models, leptoquark models, composite, two Higgs doublet models, second $Z^{\prime}$ models, anomalous Z coupling, and so on.

For heavier SUSY, if the CLM search has sufficient experimental sensitivity (such as $10^{-18}$ for MEC), it is sensitive to the SUSY mass scale up to several TeV, as shown in Fig.2. And thereby the search for CLM would be worth to carry out even if LHC does not find SUSY below TeV.

\section{Experimental}

The present experimental status and future prospect on the muon $g-2$ and the muon EDM are given in Lee Roberts's WG summary [4]. In my write-up, I focus on the experiments to search for CLM with muons [1].

First of all, why do we consider the muon system for CLM search in future? The reasons are two folds. The CLM sensitivity in the muon is the highest over the other systems, because of enormous beam intensity now $\left(\sim 10^{8} / \mathrm{sec}\right.$ at PSI $)$, and future $\left(\sim 10^{14} / \mathrm{sec}\right)$ owing to a neutrino factory frond-end R\&D. The second, the muon provides a clean test ground, on the contrary to hadrons 


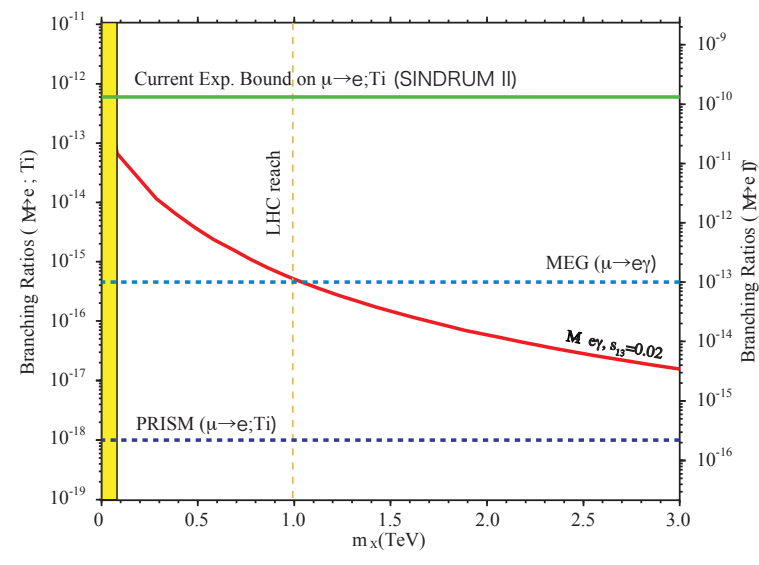

Figure 2. Prediction of the branching ratio of MEC in Ti in SUSY-Seesaw models as a function of SUSY mass scale. The PRISM (mentioned later) sensitivity is given.

where QCD corrections needed would introduces sensitivity limits.

\section{1. $\mu^{+} \rightarrow e^{+}+\gamma$ Decay}

One of the CLM process with muons is $\mu^{+} \rightarrow$ $e^{+}+\gamma$ decay. The experimental signature is simultaneous detection of a $52 \mathrm{MeV} e^{+}$and a 52 $\mathrm{MeV} \gamma$ moving back-to-back from stopped $\mu^{+}$ decay. Potential backgrounds are (1) physics background from radiative muon decay, $\mu^{+} \rightarrow$ $e^{+} \nu+\bar{\nu}+\gamma$ when neutrinos have small energy, and (2) accidental backgrounds, where two independent muon decays simultaneously and mimic a signal. At a high muon stopping rate, the latter background becomes more serious. The present upper limit is $1.2 \times 10^{-11}$ from the MEGA experiment. A new experiment MEG at PSI, aiming a sensitivity of $10^{-13}$, is under preparation. It will use a liquid-xenon photon calorimeter and cylindrical wire chambers with a graded magnetic field. The MEG experiment would start an engineering run in 2006.

\subsection{Muon-to-electron Conversion Process}

Another important CLM process with muons is a muon-to-electron conversion (MEC) process, $\mu^{-}+N(A, Z) \rightarrow e^{-}+N(A, Z)$, where at its $1 s$ state in a muonic atom, a $\mu^{-}$is captured by a nucleus, and emits an electron but not a neutrino.
The signature is a single electron with energy of the muon mass (minus the bounding energy of $\mu^{-}$in its muonic atom), of about $100 \mathrm{MeV}$. Backgrounds are an $e^{-}$from decays of bound muons at their $1 s$ state, radiative pion capture, radiative muon capture, beam electrons, and cosmic rays. The current upper limits of $\mathrm{MEC}$ at $\mathrm{Ti}$ is $6 \times 10^{-13}$ from SINDRUM-II at PSI. A new experiment MECO at BNL aiming at $10^{-16}$ was proposed but it has now been unfortunately cancelled in August, 2005.

\subsection{Future of CLM search with muons}

To aim further improvements in CLM searches, how can we proceed? The following is a summary on the constraints and conditions of various CLM searches.

\begin{tabular}{lll}
\hline Modes & Limitation & Beam \\
\hline$\mu^{+} \rightarrow e^{+} \gamma$ & detector & continuous \\
$\mu^{+} \rightarrow e^{+} e^{+} e^{-}$ & detector & continues \\
$\mu^{-} N \rightarrow e^{-} N$ & beam & pulsed \\
\hline
\end{tabular}

For the first two, accidental background is the most important. To suppress accidental backgrounds, improvement of detector resolution is critical. It is however known to be very difficult to improve further, since these involve detection of low energy photons and electrons. On the other hand, for the third one (MEC), no accidental background exists (because of single measurement), but rather beam quality should be improved. It is generally believed that by using various ideas and technologies from neutrino-factory front-end R\&D, improvement of the muon beam could be possible.

4.3.1. Required Beam Conditions for MEC The beam requirements for a future search for MEC are given as follows.

(1) High beam intensity: $\quad>10^{11} \mu^{-} / \mathrm{s}$

(2) Pulsed beam:

(3) Narrow energy spread: thin muon target

(4) High purity:

no pions in a beam

For (2), detection of the $e^{-} \mathrm{s}$ from MEC, whose time distribution follows a muon lifetime in a muonic atom, should be done between beam 
pulses so as to remove direct beam backgrounds. Regarding (3), resolution of detection of the $e^{-} \mathrm{s}$ from the MEC is determined by a thickness of the muon stopping target. To allow using a thin muon stopping target, beam energy spread should be as narrow as possible. For (4), pion radiative capture is one of the most critical backgrounds, and therefore pions in a beam should be removed as much as possible, down to $10^{-18}$ or so.

\section{Muon Factory}

The front end of a neutrino factory would produce muons of about $10^{14} / \mathrm{sec}$. That beam intensity is far beyond the beam intensity presently available, such as PSI, of $10^{8} / \mathrm{sec}$. It is quite important to consider a future experimental facility of muons at the front-end of neutrino factory. I like to call it a "Muon Factory". Each of the intense slow muon programs has specific requirements such as time structure of a beam. Therefore, special dedicated care on the facility should be considered. Also, even before the realization of a neutrino factory, the ideas and technology developed can be used to achieve beam requirements of near future muon experiments, such MEC.

\subsection{PRISM}

A project to produce a high-intensity highluminosity muon beam source is being prepared in Japan. It is called the PRISM project [5], where PRISM stands for Phase Rotated Intense Slow Muon source. The phase rotation is a technique to make beam energy spread narrower by accelerating slow muons and decelerating fast muons by high RF electric fields. PRISM consists of solenoid pion capture, transport solenoid, a muon storage ring to carry out phase rotation. The muon storage ring is a fixed field alternating gradient (FFAG) machine, which has large acceptance in transverse and longitudinal. The primary goal of PRISM is to search for the MEC at a sensitivity of less than $10^{-18}$. The beam requirements from (1) to (4) for MEC are sufficiently met by PRISM. In particular, in several turns in the FFAG muon storage ring, pions in a beam decay out and the residual fraction would be down to $10^{-18}$. A preliminary design of a detector for the MEC is made (PRIME). The layout of PRISM and PRIME is shown in Fig.3. The PRISM-FFAG ring is under construction.

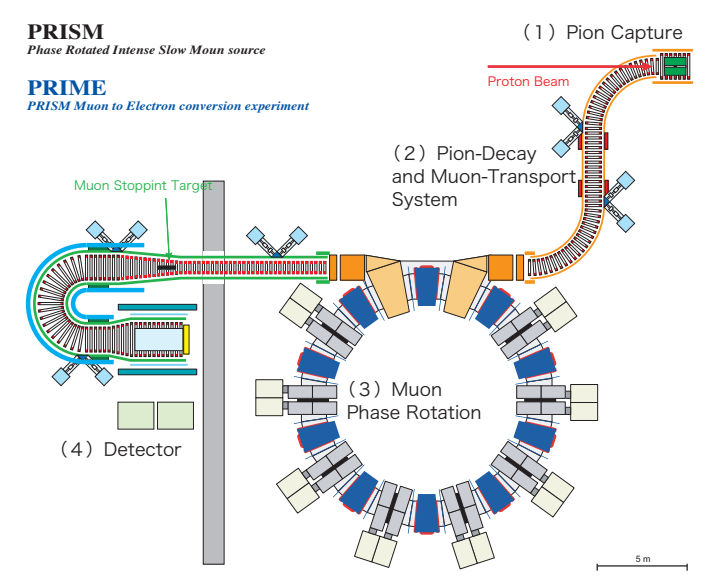

Figure 3. Layout of PRISM/PRIME. It consists of solenoid pion capture, muon transport system, muon phase rotation system based on FFAG ring, and a PRIME detector.

\section{Summary}

Physics motivation of the muon trio, muon $g-2$, muon EDM, and CLM with muons, are described, with emphasis that the front end of a neutrino factory would give great opportunity to carry out such important physics topics. The PRISM project has been launched in Japan to create a muon source of high intensity, high luminosity and high purity to search for MEC.

\section{REFERENCES}

1. Y. Kuno and Y. Okada, Rev. Mod. Phys. 73 (2001) 151-202, and references therein.

2. H. N. Brown et al., Phys. Rev. Lett. 92 (2004) 161802.

3. J.L. Feng, K.T. Matchev, Y.Shadmi, Nucl. Phys. B613 (2001) 366; PL B555 (2003) 89.

4. B.L. Roberts, in this proceedings, 2005.

5. Y. Kuno et al., J-PARC LOI, "The PRISM Project - A Muon Source of the World Highest Brightness by Phase Rotation -", 2003. 